\title{
Der Fachbuchhandel auf Ihrem Schreibtisch - Bücher online bestellen
}

\author{
Natürlich ist für viele Menschen eine Stunde, die man in einer schö- \\ nen Buchhandlung nach den neusten Titeln schmökert, ein Genuss. \\ Doch manchmal soll es einfach schnell gehen.
}

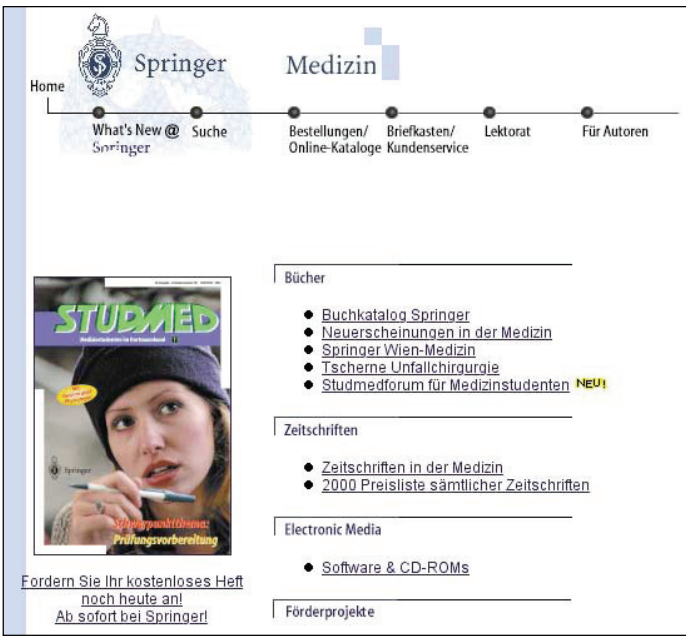

So präsentiert sich der wissenschaftliche Springer-Verlag im Internet unter den Online-Büchershops gehören darüber hinaus:

\section{- www.buecher.de \\ — www.buch.de \\ — www.buchkatalog.de \\ Online-Plattformen des Fachbuchhandels}

Der Lehmanns Online-Bookshop www.lob.de bietet in der Rubrik Medizin unter anderem eine FachliteraturSuchmaske und medizinische Fachbuchkataloge. Mit der LehmannsKreditkarte lässt sich in der „Knowledge Finder Medline" recherchieren.

Die beiden Online-Bookshops www.minerva.de und www.buchmueller.de bieten für Ärzte diverse Sonderrubriken. Darüber hinaus baut beispielsweise die Würzburger akademische Buchhandlung www.knodt.de gerade einen zusätzlichen MedizinLink-Service auf.

\section{Fachbücher direkt von der Quelle: Medizinverlage}

Natürlich präsentieren sich auch die einzelnen Verlage im Netz und bieten häufig die Möglichkeit zur direkten Bestellung ihrer Angebote. Beim Springer-Verlag unter www.springer.de/medic-de/index.html findet sich eine übersichtliche Site, wo man nach Fachgebieten suchen oder eine detaillierte Suchmaske benutzen kann. Auch der Thieme-Verlag präsentiert sich mit einer guten Katalogisierung nach Fachgebieten unter www.thieme.de. Der medizinische Fachverlag Urban \& Fischer, entstanden aus den beiden medizinischen Verlagen Gustav Fischer und Urban \& Schwarzenberg, findet sich unter www.urbanfischer.de.

Gemeinsam ist diesen Verlagen, dass sie auf der "Medizinplattform“ www.multimedica.de mit einer umfassenden Sammlung von Publikationen vertreten sind.
D ei der Suche nach Büchershops im Internet führt zunächst kein Weg an den bekannten „Top Five“ vorbei. $\mathrm{Zu}$ diesen gehört der OnlineBüchershop der Bertelsmann-Gruppe: www.bol.de. Er feierte jüngst sein einjähriges Jubiläum.

Als Newcomer kann er sich über einen beeindruckenden Senkrechtstart freuen: Seine klare Gestaltung und Benutzerführung werden Sie zu schätzen wissen. Als Mediziner empfiehlt sich die Verwendung der Suchdatenbank in der Rubrik „Bücher/Themenüberblick/Wissenschaft\&Technik/Medi zin\&Pharmazie“.

Überwiegend populär ausgerichtet war bislang das Angebot an medizinischen Büchern bei Amazon www.amazon.de. Seit kurzem steht auch dort im Bereich „Bücher/Fachbücher" eine eigene Auswahl zur Medizin zur Verfügung; ebenso gibt es für Fachleute die Rubrik „Software“. Hier werden 50 CD-ROMs vom Pschyrembel und anderen Lexika bis hin zum ICD-10 angeboten. Zu den „Großen“

\section{Das Schicksal jüdischer Dermatologen}

D ie von „Dialogpartner Öffentlichkeitsarbeit" geförderte Publikation stellt die Bedeutung der jüdischen Dermatologen Deutschlands vor der Machtübernahme durch die Nationalsozialisten 1933 und den großen Einfluss dar, den sie auf die Entwicklung der Dermatologie in ihren Emigrationsländern ausübten.

Von den 2.078 Dermatologen, die 1933 in Deutschland praktizierten, waren 589 Juden. Das entsprach einem Anteil von etwa $27,4 \%$. Im Vergleich dazu lag der Anteil jüdischer Ärzte an der Gesamtzahl der Ärzte in Deutschland bei ca. 16\%. Zu 432 jüdischen Dermatologen Deutschlands konnten Schicksals-klärende Informationen gefunden werden.

Unter den 10\% jüdischer Dermatologen, die in Konzentrationslagern der Nationalsozialisten ermordet wur- den, befanden sich so bekannte Namen wie Karl Herxheimer und Abraham Buschke. 4,4\% der jüdischen Dermatologen überlebten die NS-Zeit in Deutschland. Fast die Hälfte der jüdischen Dermatologen emigrierte, wobei Hauptemigrationsziel die USA waren. Von ihnen gingen unschätzbare Impulse auf die Entwicklung der Dermatologie in den USA aus. Unter den USA-Emigranten treten Namen wie Hermann Pinkus, Rudolf Baer, Walter Lever, Stephan Epstein, Kurt Wiener oder Alfred Hollander hervor.

Eppinger S, Vorwort von Spiegel P
Das Schicksal der jüdischen Dermatolo-
gen Deutschlands in der Zeit des
Nationalsozialismus
Dissertation, Uni Dresden 2000
Frankfurt: Mabuse-Verlag.
380 Seiten, 58 DM
ISBN 3-933050-75-8

